\title{
Contact-free measurement of respiratory rate using infrared and vibration sensors
}

\author{
Fatih Erden $^{\mathrm{a}, *}$, Ali Ziya Alkar ${ }^{\mathrm{b}}$, Ahmet Enis Cetin ${ }^{\mathrm{c}}$ \\ ${ }^{a}$ Department of Electrical and Electronics Engineering, Atllim University, Ankara 06836, Turkey \\ ${ }^{\mathrm{b}}$ Department of Electrical and Electronics Engineering, Hacettepe University, Ankara 06800, Turkey \\ ${ }^{\mathrm{c}}$ Department of Electrical and Electronics Engineering, Bilkent University, Ankara 06800, Turkey
}

\section{H I G H L I G H T S}

- A multi-modal system for respiratory rate measurement is described.

- The multi-modal system consists of two PIR sensors and a vibration sensor.

- An error of less than 2 breathings/min is achieved for different lying positions.

- Fusion of the data of different types of sensors provides a robust respiratory monitoring.

- The multi-modal system is capable of detecting sleep apnea as well.

\section{A R T I C L E I N F O}

\section{Article history:}

Received 25 August 2015

Available online 14 September 2015

\section{Keywords:}

Average magnitude difference function

(AMDF)

Empirical mode decomposition (EMD)

PIR sensor

Respiratory rate

Vibration sensor

Wavelet transform

\begin{abstract}
A B S T R A C T
Respiratory rate is an essential parameter in many practical applications such as apnea detection, patient monitoring, and elderly people monitoring. In this paper, we describe a novel method and a contact-free multi-modal system which is capable of detecting human breathing activity. The multimodal system, which uses both differential pyro-electric infrared (PIR) and vibration sensors, can also estimate the respiratory rate. Vibration sensors pick up small vibrations due to the breathing activity. Similarly, PIR sensors pick up the thoracic movements. Sensor signals are sampled using a microprocessor board and analyzed on a laptop computer. Sensor signals are processed using wavelet analysis and empirical mode decomposition (EMD). Since breathing is almost periodic, a new multi-modal average magnitude difference function (AMDF) is used to detect the periodicity and the period in the processed signals. By fusing the data of two different types of sensors we achieve a more robust and reliable contact-free human breathing activity detection system compared to systems using only one specific type of sensors.
\end{abstract}

(C) 2015 Elsevier B.V. All rights reserved.

\section{Introduction}

Respiratory rate is an important vital sign in assessing the physical and psychological health of human beings. Respiratory rate, especially the expiratory time is a good predictor of the level of individual anxiety [1]. It is reported in [2] that a serious increase in the breathing activity is the most important indicator of cardiac arrest in hospital wards. It is also known that many lung and heart diseases such as pneumonia affect respiratory rate [3]. Therefore respiratory monitoring is a crucial tool in intensive care units, hospitals, elderly care units, and home medical care services.

Systems for respiratory monitoring can be classified into two categories: (i) systems which are worn on the human's body, e.g. thoracic impedance pneumography, photoplethysmography, and

\footnotetext{
* Corresponding author. Tel.: +90 312586 8254; fax: +90 3125868091

E-mail address: fatih.erden@atilim.edu.tr (F. Erden).
}

(ii) systems which measure the human's near-environment, e.g. sound recording, carbon dioxide sensing. The work in [4-7] are good examples of the first group. Carmo and Correia [4] use a wireless electronic shirt with embedded sensors to measure the heart rate and respiratory frequency. An electromagnetic biosensor centered on the subject's sternum is used for respiratory monitoring in [5]. Peng et al. [6] propose a system with video, pyro-electric infrared (PIR) sensors and wearable actigraphy based monitoring system for sleep monitoring. However it fails to detect the period of breathing and PIR sensors are only used in on/off mode. In [7] a wireless wearable bioimpedance device is developed. But the device basically aims to detect the abnormal breathing waveforms in the bioimpedance signal and cannot estimate the respiratory rate accurately.

The studies in [8-14] exemplify the second group. In [8,9], radar sensor based systems for central apnea detection and respiratory monitoring are described, respectively. A biosignal measurement 
system, which measures the air pressure in an air mattress, to monitor the heartbeat and respiration of a person lying in bed is introduced in [10]. Wang et al. [11] propose a sensor based sleep monitoring system to assess the quantity and quality of sleep for elderly people. But it uses PIR sensors in on/off mode only and does not determine the period of breathing. They also use bed sensors but not for detection of the breathing activity.

Infrared camera based imaging systems are also used to monitor the respiratory activity [12]. Wai et al. [13] describe an array of bed sensors and cameras to monitor client position in bed and different situations when the client is out of bed. While the article describes a bed monitoring system to detect sleep patterns, the system focuses on monitoring falls but not the breathing activity. Furthermore, the use of cameras in bedroom raises issues related to personal privacy of the individual.

In [14], a system including only PIR type sensors to monitor the respiratory movements is presented. Fourier analysis of the sensor signals are used to find out the abnormalities, in particular sleep apnea, in a patient's breathing activity. Respiratory rate measurement is mentioned as a further investigation in the study. In [14], analog signal acquisition from the PIR sensors is not described. In Section 2, we present a circuit structure developed for analog data acquisition from a PIR sensor.

In this paper, we describe a novel contact-free human breathing activity detection system using a plurality of sensors. The system is also capable of estimating the respiratory rate. In contrast to the single-sensor type systems, our multi-modal system consists of two types of sensors: PIR and vibration sensors. PIR sensors are placed onto a stand near the bed and the vibration sensor is placed on the bedding frame. A typical graphical description of the multimodal system setup is shown in Fig. 1. Vibration sensors pick up small vibrations due to breathing activity. Similarly, PIR sensors pick up the thoracic movements. Sensor signals are sampled using a microprocessor board and processed using wavelet analysis and empirical mode decomposition (EMD) on a laptop computer. Our data analysis is different from [14]. We process the sensor signals using wavelet analysis. Since breathing is almost periodic, average magnitude difference function (AMDF) is used to detect the periodicity. We also define a novel AMDF by fusing the PIR and vibration sensors' signals. By using two different types of sensors we achieve a more robust and reliable contact-free human breathing activity detection system compared to systems using only one specific type of sensors.

The paper is organized as follows: Operating principles of the sensors and data acquisition from the sensors are described in Section 2. Wavelet analysis and EMD based sensor data processing is described in Section 3. Section 4 reports the results of experimental validation and Section 5 concludes the paper.

\section{Data acquisition}

\subsection{PIR sensors}

PIR sensors are widely used commercially for motion detection, e.g. in automation of electrical appliances [15], design and implementation of a home embedded surveillance systems [16]. These applications are, in general, based on the on/off decisions of the PIR sensors.

A differential PIR sensor basically measures the difference of infrared radiation density between the two pyro-electric elements $\left(\mathrm{IR}_{1}, \mathrm{IR}_{2}\right)$ inside. Fig. 2 shows the block diagram of a typical differential PIR sensor, $\left(s_{1}\right)$ and $\left(s_{2}\right)$ are the outputs of the pyro-electric elements and $(\mathrm{g})$ is ground. Normal temperature alterations and changes caused by airflow are cancelled by the two elements connected in parallel. If these elements are exposed to the same

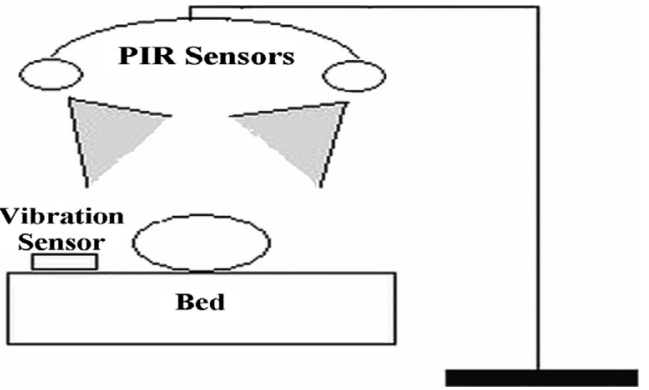

Fig. 1. A typical illustration of the contact-free multimodal human breathing activity detection system consisting of two PIR sensors and a vibration sensor.

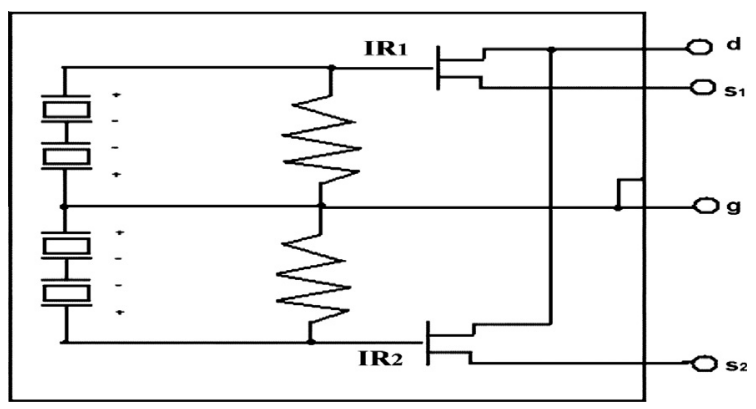

Fig. 2. Model of the inner structure of a differential PIR sensor

amount of infrared radiation, they cancel each other and the sensor produces a zero-output at (d).

Commercially available PIR motion detector circuits produce binary outputs. However, it is possible to capture a continuoustime analog signal representing the amplitude of the voltage signal which corresponds to the transient behavior of the circuit. We use a circuit, which we developed for flickering flame detection [17], to capture an analog output signal from the PIR sensor and it is shown in Fig. 3. The circuit consists of four operational amplifiers (op amps), U1A, U1B, U1C and U1D. U1A and U1B constitute a two stage amplifier circuit whereas U1C and U1D couple behaves as a comparator. The very-low amplitude raw output at the 2 nd pin of the PIR sensor is amplified through the two stage amplifier circuit. The amplified signal at the output of U1B is fed into the comparator structure which outputs a binary signal, either $0 \mathrm{~V}$ or $5 \mathrm{~V}$ in standard motion detectors. Instead of using the binary output in the original version of the PIR sensor read-out circuit, the analog output signal at the output of the 2 nd op amp U1B is captured.

The analog output signal is digitized using a microcontroller with a sampling rate of $20 \mathrm{~Hz}$ and transferred to a general-purpose laptop computer for further processing. A typical respiratory rate for a healthy adult at rest is $12-20$ breaths per minute. Average resting respiratory rates vary with age. Infants take $30-60$ breaths per minute. Therefore even a $2 \mathrm{~Hz}$ sampling frequency is enough for digitization of the analog sensor signal due to Shannon's sampling theorem. However a higher sampling rate $(20 \mathrm{~Hz})$ is selected for digitization. A typical differential PIR sensor signal due to a human breathing at a $1 \mathrm{~m}$ distance from the sensor is shown in Fig. 4. It is clear from the figure that the signal is almost periodic.

\subsection{Vibration sensor}

Vibration sensors are usually based on accelerometers which are used to detect seismic activity, vibrations in mechanical systems and machines, buildings, and they are also used in 


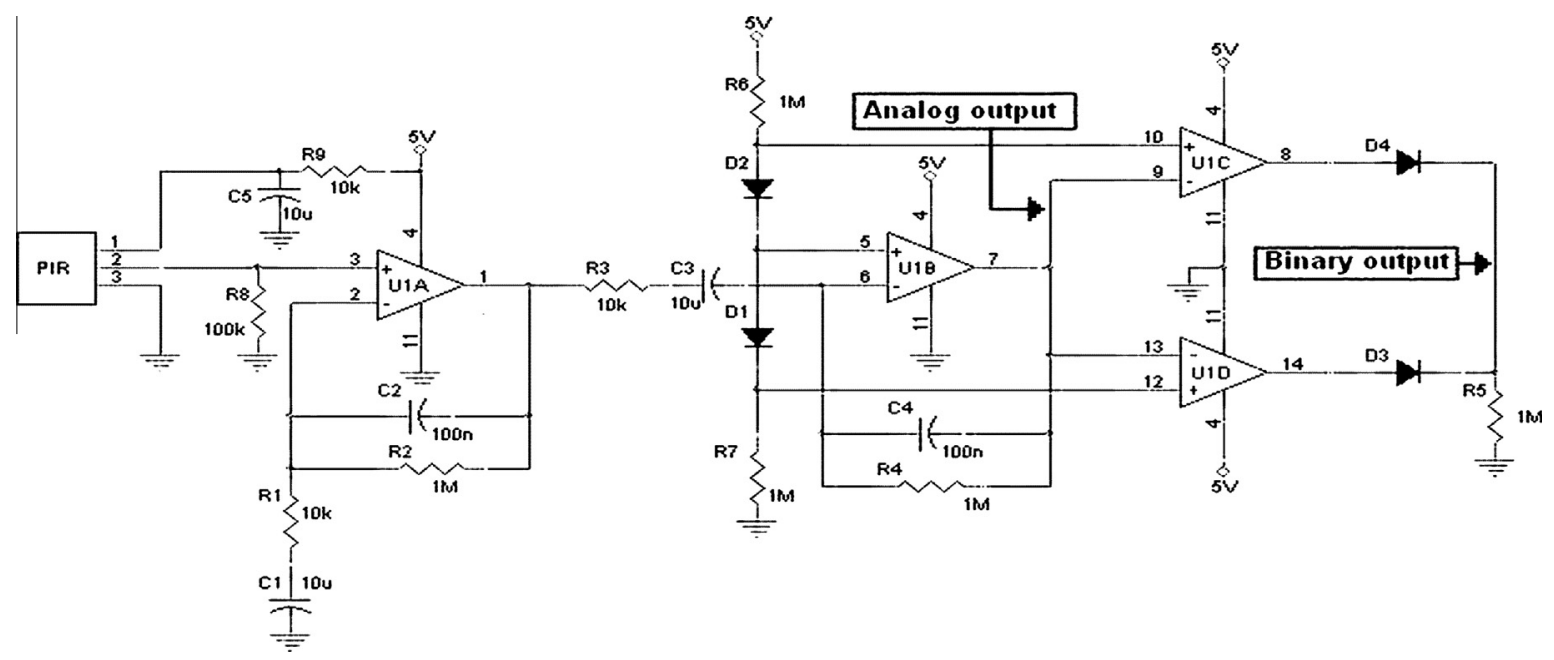

Fig. 3. The diagram of the circuit for capturing an analog output signal from a PIR sensor.

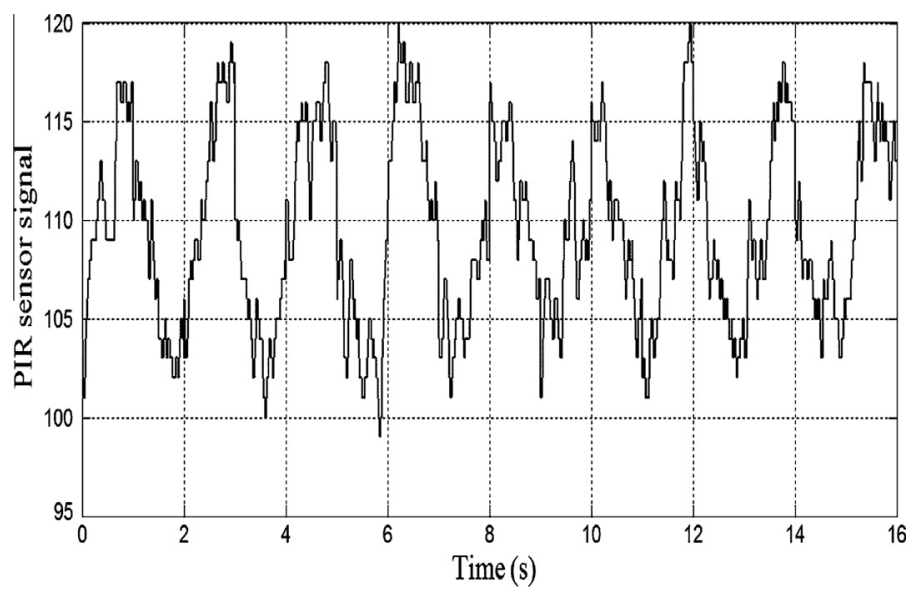

Fig. 4. A typical PIR sensor signal due to a human breathing at a $1 \mathrm{~m}$ distance from the sensor. Sampling frequency is $20 \mathrm{~Hz}$.

cell-phones and tablet computers. In commercial accelerometers, piezo-electric, piezo-resistive and capacitive components are used to convert the mechanical motion into an electrical signal. Electrical signal obtained from the vibration sensor is sampled at $20 \mathrm{~Hz}$, which is sufficient enough to pick up the vibrations in the bedding frame due to breathing activity, converted into a digital form by a microprocessor, and then transformed to a general-purpose laptop computer for discrete-time processing.

The vibration sensor used in this work provides acceleration information in three axial ( $x$-roll, $y$-pitch, and $z$-yaw) directions. During recordings of the vibration sensor signal, it is observed that the acceleration information coming from the $z$-yaw axis is not consistent with the breathing activity, but the information in $x$-roll and $y$-pitch axes are. Therefore only the outputs in $x$-roll and $y$-pitch axes are utilized. To make the vibration sensor output comparable with the PIR sensor output, we denote it by a single representative signal $\sqrt{x^{2}+y^{2}}$, where $x$ and $y$ represent the signal values in $x$-roll and $y$-pitch axes, respectively. A typical vibration sensor signal due to a human breathing activity is shown in Fig. 5. The representative output signal of the vibration sensor also exhibits an almost periodic waveform. When a person rolls on his/her bed, the vibration sensor picks up a large amplitude signal shown in Fig. 6 which is clearly not a periodic signal.

\section{Data processing}

To detect the breathing activity and estimate the respiratory rate, sensor signals are first processed using two different methods: (i) wavelet decomposition and (ii) EMD. Then the periodicity of the sensor signals, if exits, is detected using the AMDF.

\subsection{Wavelet decomposition}

The human breathing activity is a low-frequency activity with the highest frequency of $1 \mathrm{~Hz}$. Therefore the sampling frequencies of the sensors' output signals should be at least twice that of the highest frequency, as they already are. In addition, the noise is usually a high-frequency activity. Since we are dealing with the almost periodic breathing activity, we reduce the frequency content of the recorded sample signals inside the band of interest using a wavelet tree.

Wavelet-domain analysis provides robustness to variations in the sensor signal caused by temperature and airflow changes in the environment. A wavelet stage consists of a high pass filter and a low-pass filter and downsamplers. A wavelet tree, which consists of two or more wavelet stages, decomposes the input signal into subsignals with a specific frequency content. For example, 


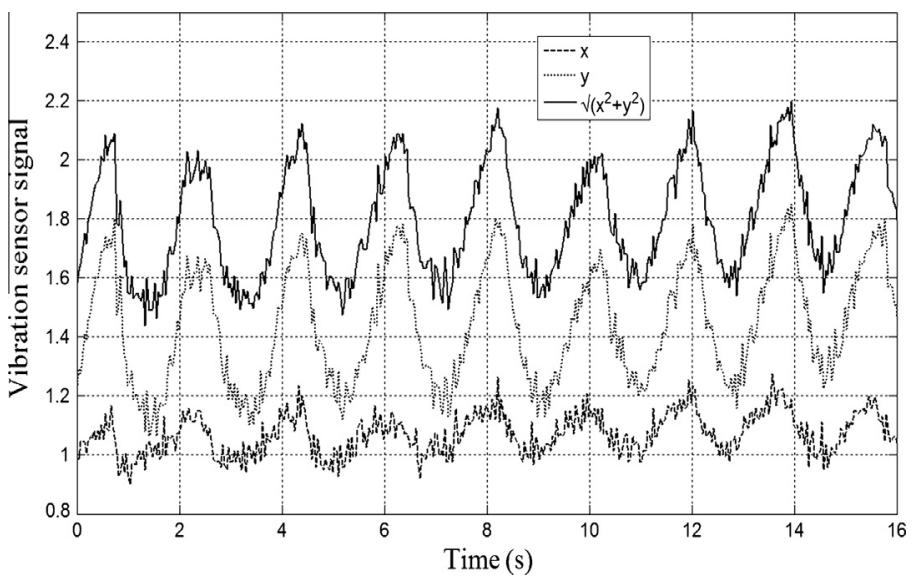

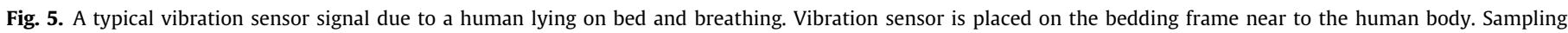
frequency is $20 \mathrm{~Hz}$

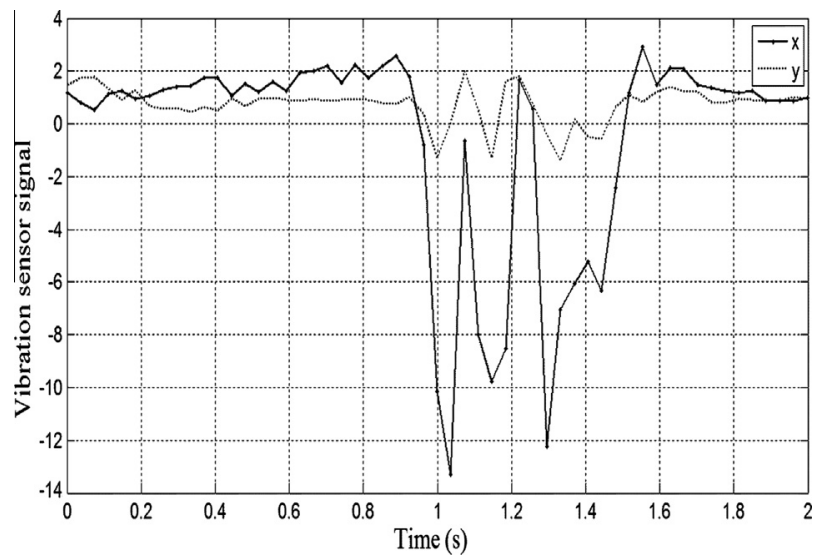

Fig. 6. A $2 \mathrm{~s}$ vibration sensor output signal due to a person rolling on bed.

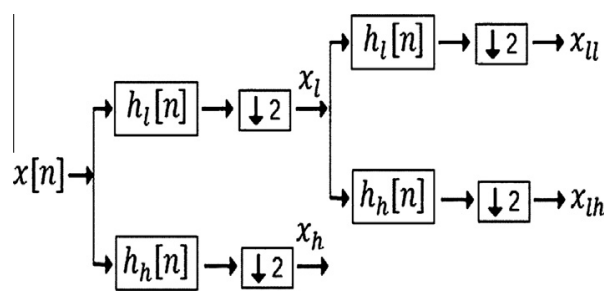

Fig. 7. A 2 stage wavelet decomposition structure.

after a single stage decomposition in the wavelet tree in Fig. 7 the subsignal $x_{l}$ comes from the $(0-10 \mathrm{~Hz})$ band of the original sensor signal which is sampled at $20 \mathrm{~Hz}$. The wavelet signal $x_{h}$ comes from the $(10-20 \mathrm{~Hz})$ band of the original signal therefore we simply ignore it. Since breathing activity is a low-frequency activity, we further process $x_{l}$ using the second stage of the wavelet tree and we obtain $x_{l l}$ which comes from the $(0-5 \mathrm{~Hz})$ band. The subsignal $x_{l h}$ is useless again. Decimation by 2 in each wavelet stage halves the time resolution. Therefore, in the wavelet-domain each $N$ samples correspond to $m$ seconds, where $m$ is the number of stages in the wavelet decomposition tree, whereas each $N$ samples correspond to $1 \mathrm{~s}$ in the original sensor data. Number of the wavelet stages depends on the sampling frequency and the desired frequency content. Here we use a 4 stages wavelet tree to have only the $(0,1 \mathrm{~Hz})$ band information. We use the Lagrange wavelet in which the low-pass and the high-pass filters are

$h_{l}[n]=[1 / 4,1 / 2,1 / 4]$

and

$h_{h}[n]=[1 / 4,-1 / 2,1 / 4]$,

respectively [18]. Lagrange wavelet is the most computationally efficient wavelet since it can be implemented without any multiplications. It is also more efficient than Fourier analysis for this problem because of the same reason. Other wavelet filterbanks mentioned in the literature can be also used for wavelet analysis of sensor signals. The wavelet transform of one of the PIR sensors and the vibration sensor output signals due to the human breathing activity are shown in Fig. 8. The wavelet transform, while reducing the computational cost, preserves the periodic behavior of the signals and it is obvious from the figure that there is a strong correlation between the PIR and the vibration sensor wavelet signals due to the human breathing activity.

\subsection{Empirical mode decomposition (EMD)}

EMD method, which is introduced by Huang et al. [19], is used to adaptively decompose a signal into intrinsic oscillations. Because of the simplicity and the efficiency of its algorithm, the EMD is widely used in several fields including biomedical applications $[20,21]$. Both the EMD and the wavelets decompose a signal into different frequency bandwidths. The main difference between them is that the EMD decomposes a signal adaptively and is fully data-driven, whereas the wavelet decomposition uses some pre-defined filters.

The EMD method starts by estimating a signal as a sum of a low-frequency and a high-frequency components. The highfrequency component, which is referred to as detail, is obtained by subtracting the low-frequency part of the signal from the original signal. The same procedure is then applied to the low-frequency part considering it as a new signal to extract a new detail. Given a signal $x(t)$, the EMD algorithm can be summarized as follows [19]:

1. Find all maxima and minima of $x(t)$.

2. Interpolate between minima (maxima) points separately using a cubic spline to obtain the lower (upper) envelope, $e_{\min }(t)$ $\left(e_{\max }(t)\right)$.

3. Compute the low-frequency component, $m(t)=\left(e_{\min }(t)+\right.$ $\left.e_{\max }(t)\right) / 2$. 


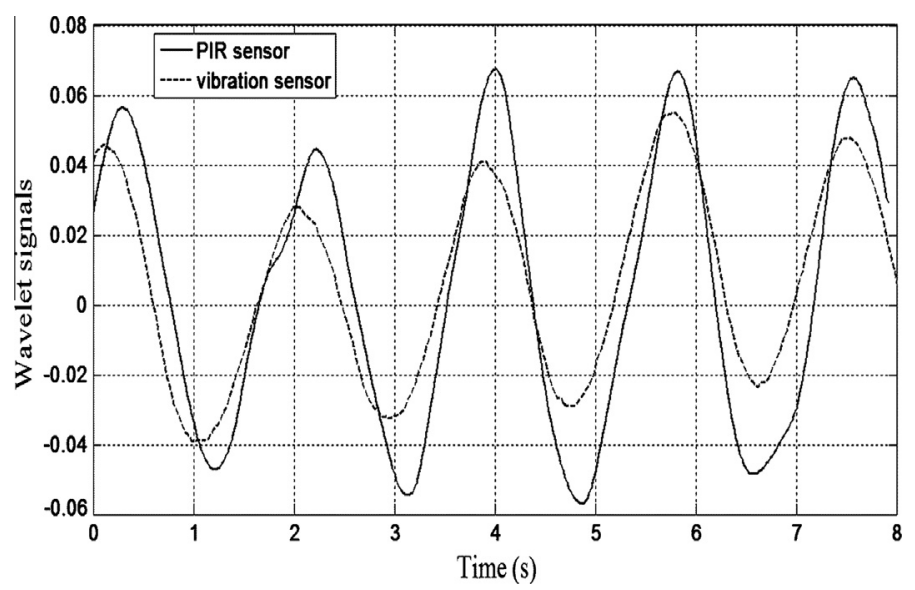

Fig. 8. Wavelet signals of the PIR and the vibration sensors due to human breathing activity.

4. Extract the detail, $d(t)=x(t)-m(t)$.

5. Iterate on $m(t), x(t)=m(t)$.

Under the zero-mean condition, the detail $d(t)$ is referred to as an Intrinsic Mode Function (IMF). The above procedure is repeated until the IMF definition is met. The output of the EMD of the PIR sensor signal due to a human breathing at a $1 \mathrm{~m}$ distance from the sensor is shown in Fig. 9. The IMF definition is met after 6 iterations of the algorithm, i.e., the dashed line in Fig. 9 is an IMF. Each oscillation of the IMF corresponds to one breathing activity.

\subsection{Periodicity detection using the AMDF}

The EMD and the wavelet-decomposed signals are then processed using the average magnitude difference function (AMDF) to detect the periodic behavior. The AMDF is widely used in speech processing for voiced phoneme detection and to determine the period of voiced sounds. The AMDF is defined as follows:

$\operatorname{AMDF}[k]=1 / N \sum_{n}|u[n]-u[n-k]|$,

where $u[n]$ represents the wavelet signal $x_{l^{4}}[n]$ which comes from the $(0-1.25 \mathrm{~Hz})$ band of the original signal or the IMF and $N$ is the number of samples within the analysis time-window. In this case, the analysis time-window is $1 \mathrm{~min}$, since we desire to determine the respiratory rate in terms of breathing/min. Output of the AMDF for the PIR sensor wavelet signal in Fig. 8 is plotted in Fig. 10. AMDF

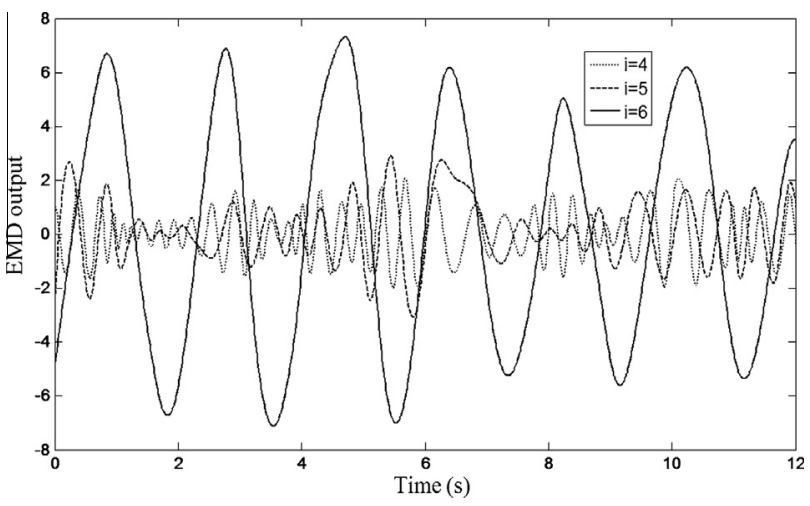

Fig. 9. Output of the EMD of the PIR sensor signal due to a human breathing activity (for 4,5 , and 6 iterations). exhibits a set of local minima corresponding to the periods of $u[n]$. We detect the first local minimum and based on the time index of the minimum we determine the period which in turn gives the respiration rate. For example, the person whose AMDF data shown in Fig. 10 takes a breath approximately in every $1.84 \mathrm{~s}$. Periodicity can also be detected from the wavelet signals shown in Fig. 8 and the IMF in Fig. 9. However, AMDF provides robustness because of data averaging in (3). Furthermore, it can be computed without performing any multiplications.

We further define a novel AMDF function by fusing the two sensor signals as follows:

$\operatorname{AMDF}_{2}[k]=1 / N \sum_{n} \alpha_{u}|u[n]-u[n-k]|+\alpha_{v}|v[n]-v[n-k]|$,

where $u[n]$ is the wavelet signal or the IMF of one of the PIR sensors, $v[n]$ is the wavelet signal or the IMF of the vibration sensor (or the other PIR sensor), and $\alpha_{u}$ and $\alpha_{v}$ are some weights. If there is the plurality of sensors, (4) may be arranged as follows:

$\operatorname{AMDF}_{L}[k]=1 / N \sum_{i=1}^{L} \sum_{n} \alpha_{i}\left|u_{i}[n]-u_{i}[n-k]\right|$,

where $L$ is the number of sensors. We name the $\mathrm{AMDF}_{2}$ function the multimodal AMDF because it uses a plurality of sensor signals to determine the periodic or aperiodic nature of the recordings. Obviously, when there is periodicity in either one of the recorded signals, the $\mathrm{AMDF}_{2}$ will exhibit a local minimum. This is important because one or more of the sensors may not measure a meaningful signal due to the sleeping position of the person. Since the human body has to move somewhat during breathing, either the vibration sensor or one of the PIR sensors will pick up the breathing activity and the multimodal AMDF will produce a local minimum corresponding to the respiratory rate. Another advantage of the $\mathrm{AMDF}_{2}$ is that it can handle phase differences between the sensors' signals which may otherwise result in an inaccurate detection of the respiratory rate.

\section{Experiments and results}

Our experimental multi-modal respiratory rate detection system consists of two differential PIR sensors placed onto a stand near the bed and a vibration sensor placed on the bedding frame. PIR sensors are aligned with the human's chest as shown in Fig. 1. The vibration sensor does not need to contact the human body. 


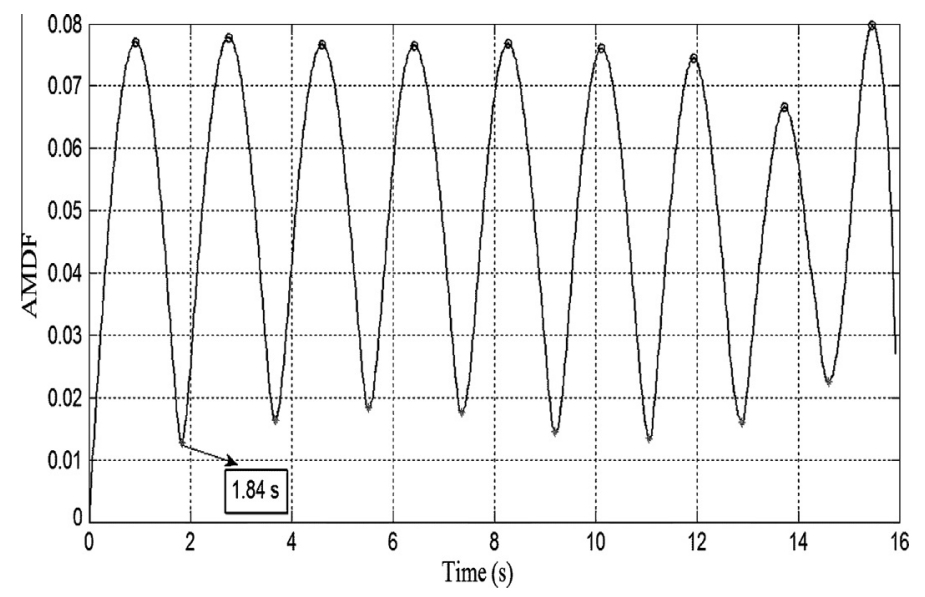

Fig. 10. Output of the AMDF for the PIR sensor wavelet signal in Fig. 8.

Table 1

Reliability percentages of respiratory rate measurements of the multi-modal system for 10 subjects.

\begin{tabular}{lllc}
\hline Subject position & $|\varepsilon|<0.5$ & $0.5<|\varepsilon|<1$ & $1<|\varepsilon|<2$ \\
\hline Wavelet method & & & \\
Supine (\%) & 73 & 21 & 6 \\
Prone (\%) & 64 & 28 & 8 \\
Side-lying (\%) & 58 & 32 & \\
EMD method & & & 70 \\
Supine (\%) & 71 & 22 & 5 \\
Prone (\%) & 66 & 29 & 9 \\
Side-lying (\%) & 58 & 33 & \\
\hline
\end{tabular}

$\varepsilon$ : the error (breathing/min) in the respiratory rate measurement.

The reliability percentages of the respiratory rate measurements for 10 different subjects between ages 10 and 49 using the wavelet decomposition and the EMD are presented in Table 1. The subjects are asked to lie in different positions, i.e. supine, prone and side-lying positions, and breath for one minute. When a person moves during his sleep in the bed, the vibration and PIR sensors generate large amplitude signals. Obviously, this time window is not due to the breathing activity and we do not include that portion of the sensor signals to respiration rate estimation process. AMDF does not exhibit a local minimum for such windows. During the experiments, the stand holding the PIR sensors is set to be at various distances from $20 \mathrm{~cm}$ to $1 \mathrm{~m}$ from the subject. Experiments include an average of 30 recordings for each subject in each lyingposition and totally more than 900 recordings. We observed that no recordings differ more than 2 breathings/min from the ground truth. In addition, if the accuracy is defined such that the measurement error is less than 1 breathing/min, the multi-modal system using wavelet decomposition achieves an average accuracy rate of $92 \%$ in the respiratory rate measurements. The average accuracy rate obtained by using the EMD is $93 \%$. The EMD method performs slightly better. But the computational complexity of the EMD algorithm is significantly greater, due to the interpolation operation and also the repetition of the algorithm for several times.

The PIR-only and the vibration-only systems are able to estimate the respiratory rate separately. But in some lying positions, in particular prone position for the PIR sensors and side-lying position for the vibration sensor, sensors mostly fail to detect the breathing activity. But the proposed system can handle this problem by its multi-modal structure. As Table 1 reports, the system does not miss any breathing activity due to the sleeping position of a person.

When a person has a sleep apnea, he or she does not move therefore the wavelet signals and the IMFs corresponding to both vibration sensor and the PIR sensors become very close to zero. As a result the sleep apnea can be also detected by the proposed system. In this case the AMDF function does not have a local minimum in the apnea windows. Its value increases as $k$ increases. One such instance is shown in Fig. 11. Consequently the AMDF function can be used to detect sleep apnea as well.

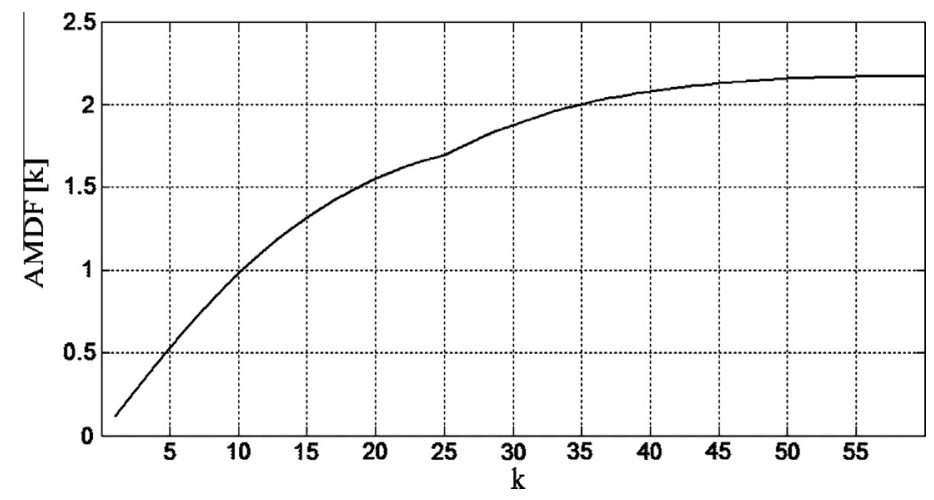

Fig. 11. Output of the AMDF for the vibration sensor wavelet signal due to a sleep apnea. There is no local minimum. 


\section{Conclusion}

In this paper, we proposed a novel system for a reliable contactfree measurement of the respiratory rate. The system consists of two differential PIR sensors and a vibration sensor. Up to our knowledge, this is the first study that uses the PIR and vibration sensors combination to estimate the respiratory rate. We defined a novel multimodal AMDF function to fuse the data of the multiple sensors. Some of the sensors may not measure a meaningful signal due to the sleeping position of the person. Since the person's body moves noticeably during breathing, either vibration or PIR sensors pick up the breathing activity and the multimodal AMDF function detects the periodicity and the period of breathing. The proposed multi-modal system is also able to detect the sleep apnea. We compared the performances of the wavelet and the EMD methods. The overall system can be implemented using a single low-power microprocessor since both the wavelet transform and the AMDF do not need any complicated data processing operations.

\section{Conflict of interest}

There is no conflict of interest.

\section{References}

[1] I. Homma, Y. Masaoka, Breathing rhythms and emotions, Exp. Physiol. 93 (9) (2008) 1011-1021.

[2] M.A. Cretikos, R. Bellomo, K. Hillman, J. Chen, S. Finfer, A. Flabouris, Respiratory rate: the neglected vital sign, Med. J. Aust. 188 (11) (2008) 657-659.

[3] S. Min, Y. Yun, H. Shin, Simplified structural textile respiration sensor based on capacitive pressure sensing method, IEEE Sens. J. 14 (9) (2014) 3245-3251.

[4] J.P. Carmo, J.H. Correia, RF CMOS transceiver at $2.4 \mathrm{GHz}$ in wearables for measuring the cardio-respiratory function, Measurement 44 (2011) 65-73.

[5] B. Padasdao, E. Shahhaiadar, C. Stickley, O. Boric_Lubecke, Electromagnetic biosensing of respiratory rate, IEEE Sens. J. 13 (11) (2013) 4204-4211.

[6] Y.T. Peng, C.Y. Lin, M.T. Sun, C.A. Landis, Multimodality sensor system for longterm sleep quality monitoring, IEEE Trans. Bimoed. Circuits Syst. 1 (3) (2007) 217-227.

[7] V. Seppä, J. Väisänen, P. Kauppinen, J. Malmivuo, J. Hyttinen, Measuring respirational parameters with a wearable bioimpedance device, in: Proc. 13th
International Conference on Electrical Bioimpedance and the 8th Conf. Electrical Impedance Tomography, 2007, pp. 663-666.

[8] G.P. Weinreich, P. de Chazal, Y. Wang, S. Teschler, H. Teschler, A pilot study on non-contacting detection of central apneas, in: Proc. World Congress on Medical Physics and Biomedical Engineering, 2009, pp. 790-791.

[9] D. Zito, D. Pepe, M. Mincica, F. Zito, A. Tognetti, A. Lanata, D. De Rossi, SoC CMOS UWB Pulse radar sensor for contactless respiratory rate monitoring, IEEE Trans. Biomed. Circuits Syst. 55 (6) (2011) 503-510.

[10] Y. Kurihara, K. Watanabe, Development of unconstrained heartbeat and respiration measurement system with pneumatic flow, IEEE Trans. Biomed. Circuits Syst. 6 (6) (2012) 503-510.

[11] H. Wang, H. Zheng, J.C. Augusto, S. Martin, M. Mulvenna, W. Carswell, J. Wallace, P. Jeffers, B. Taylor, K. McSorley, Monitoring and analysis of sleep pattern for people with early dementia, in: Proc. IEEE International Conference on Bioinformatics and Biomedicine Workshops, 2010, pp. 405-410.

[12] J.N. Murthy, J. van Jaarsveld, J. Fei, I. Pavlidis, R.I. Harrykissoon, J.F. Lucke, S. Faiz, R.J. Castriotta, Thermal infrared imaging: a novel method to monitor airflow during polysomnography, Sleep 32 (11) (2009) 1521-1527.

13] A.A.P. Wai, W. Huang V.F.S. Fook, J. Biswas, H. Chi-Chun, L. Koujuch, Situationaware patient monitoring in and around the bed using multimodal sensing intelligence, in: Proc. IEEE International Conference on Intelligent Environments, 2010, pp. 128-133.

[14] V. Hers, D. Corbugy, I. Joslet, P. Hermant, J. Demerteau, B. Delhougne, G. Vandermoten, J.P. Hermanne, New concept using Passive Infrared (PIR) technology for a contactless detection of breathing movement: a pilot study involving a cohort of 169 adult patients, J. Clin. Monit. Comput. 27 (5) (2013) 521-529.

[15] R. Samson, T. Winings, Automated dispenser for disinfectant with proximity sensor, U.S. Patent 5695 091, December 9, 1997.

[16] Y.W. Bai, L.S. Shen, Z.H. Li, Design and implementation of an embedded home surveillance system by use of multiple ultrasonic sensors, IEEE Trans. Consum. Electron. 56 (1) (2010) 119-124.

[17] F. Erden, B.U. Toreyin, E.B. Soyer, I. Inac, O. Gunay, K. Kose, A.E. Cetin, Wavelet based flickering flame detector using differential PIR sensors, Fire Saf. J. 53 (2012) 13-18.

[18] C.W. Kim, R. Ansari, A.E. Cetin, A class of linear-phase biorthogonal wavelets, in: Proc. IEEE International Conference on Acoustics, Speech, and Signal Processing, 1992, pp. 673-676.

[19] N.E. Huang, Z. Shen, S.R. Long, M.C. Wu, H.H. Shih, Q. Zheng, N.-C. Yen, C.C. Tung, H.H. Liu, The empirical mode decomposition and Hilbert spectrum for non-linear and non-stationary time series analysis, in: Proc. Royal Society London Series A454, 1998, pp. 903-995.

[20] C.D. Papadaniil, L.J. Hadjileontiadis, Efficient heart sound segmentation and extraction using ensemble empirical mode decomposition and Kurtosis features, IEEE J. Biomed. Health Inform. 18 (4) (2014) 1138-1152.

[21] K.T. Sweeney, S.F. McLoone, T.E. Ward, The use of ensemble empirical mode decomposition with canonical correlation analysis as a novel artifact removal technique, IEEE Trans. Biomed. Eng. 60 (1) (2013) 97-105. 